\title{
Electroweak baryogenesis and CP violation from fourth generation
}

Masaya KOHDA ${ }^{* \dagger}$

National Taiwan University

E-mail: mkohdadhep1.phys.ntu.edu.tw

We address the electroweak baryogenesis in the standard model (SM) with a 4th generation (4G), dubbed SM4. One of the reasons, that the SM fails to explain the baryon asymmetry of the universe (BAU), is insufficiency of $\mathrm{CP}$ violation arising from the Kobayashi-Maskawa (KM) phase, while the $4 \mathrm{G}$ quarks introduces new $\mathrm{CP}$ violating phases still within the $\mathrm{KM}$ framework. An intuitive argument, using a 4 generation analog of the Jarlskog invariant, suggests a huge enhancement of the $\mathrm{CP}$ asymmetry with respect to the 3 generation case [1]. Given a first-order electroweak phase transition (EWPhT), we estimate the BAU produced due to the scattering of quarks and antiquarks by the wall of an expanding bubble of Higgs condensate, by taking into account the QCD damping effects. We demonstrate that the $4 \mathrm{G}$ effect actually enhances the $\mathrm{CP}$ asymmetry as expected and it could be large enough to explain the observed baryon-to-entropy ratio, $n_{B} / s \simeq 0.9 \times 10^{-10}$.

XXIst International Europhysics Conference on High Energy Physics

21-27 July 2011

Grenoble, Rhône-Alpes France

\footnotetext{
* Speaker.

${ }^{\dagger}$ I thank the organizers for the opportunity to present a poster, and my collaborators Wei-Shu Hou and Yoshio Kikukawa.
} 
Within the SM of particle physics, a possible mechanism to explain the BAU is electroweak baryogenesis which operates during a first-order EWPhT accompanied by bubble nucleation. The SM fails, however, to explain the observed BAU due to two reasons: (i) the EWPhT is not firstorder for an experimentally allowed Higgs boson mass, (ii) CP violation from the KM phase is not sufficient [2] 3]. We show that the latter could be overcome by the addition of the $4 \mathrm{G}$ quarks.

At the heart of the SM electroweak baryogenesis, which was proposed by Farrar and Shaposhnikov (FS) [2], is CP violating scattering of quarks by the wall of an expanding bubble. CP violation is induced by thermal effects, i.e. interactions of quarks with charged $\mathrm{W}$ and Higgs bosons in a plasma, which are taken into account by employing a quasiparticle picture in the scattering problem due to the bubble wall. Although the first attempt by FS found that CP violation from the KM phase could be large enough to explain the BAU, it was refuted by subsequent analyses [3] which showed that the inclusion of the quasiparticle width (damping rate), which was not considered by FS, reduces the BAU to a negligible amount. In the following analysis, we follow the steps of Huet and Sather (HS) in [3] by extending them to the 4 generation case.

We assume that the EWPhT is strongly first-order so that the washout of the baryon asymmetry due to sphaleron processes is negligible in the broken phase. The generated BAU is, then,

$$
\frac{n_{B}}{s} \sim-10^{-2} \alpha_{W} v_{W} \frac{1}{T} \int \frac{d \omega}{2 \pi} n_{0}(\omega)\left[1-n_{0}(\omega)\right] \Delta(\omega),
$$

where $v_{W} \sim 0.1$ is the wall velocity, and $n_{0}(\omega)=1 /[\exp (\omega / T)+1]$ is the Fermi-Dirac distribution. $\Delta(\omega)$ is the $\mathrm{CP}$ asymmetry for the reflection probabilities of right-handed quarks (quasiparticles) incident from the unbroken phase with an energy $\omega$, summed over all flavors. $\Delta(\omega)$ is obtained by solving the effective Dirac equations for the quasiparticles in the presence of space-dependent mass terms. Assuming the wall of zero thickness, HS solved the equations analytically based on the Green's function method, which gives perturbative expansions for the reflection coefficients in terms of the quark mass matrices $M_{u, d}$.

The leading-order contribution to $\Delta(\omega)$ from the scattering of the d-type quarks is given by

$$
\Delta_{d}(\omega)=\frac{4}{3}\left(\frac{27 \pi \alpha_{W} T^{2}}{64 \Omega_{0} M_{W}^{2}}\right)^{3}\left[1+\left(\frac{\omega-\Omega_{0}}{\gamma}\right)^{2}\right]^{-6}\left(\frac{1}{6 \gamma}\right)^{9} \operatorname{Im} \operatorname{Tr}\left[M_{u} M_{u}^{\dagger}, M_{d} M_{d}^{\dagger}\right]^{3},
$$

where $\Omega_{0} \simeq g_{s} T / \sqrt{6}$ is the flavor independent part of the thermal masses for the quarks. $\gamma$ is the quasiparticle width and we use the one calculated in QCD, $\gamma \simeq 0.15 g_{s}^{2} T$, neglecting possible flavor dependent corrections from the Yukawa interactions. Then, the $4 \mathrm{G}$ effects enter solely through the last factor in Eq. (2). In the 3 generation case, this factor is the well-known Jarlskog invariant

$$
\operatorname{Im} \operatorname{Tr}\left[M_{u} M_{u}^{\dagger}, M_{d} M_{d}^{\dagger}\right]^{3}=6 J\left(m_{t}^{2}-m_{c}^{2}\right)\left(m_{t}^{2}-m_{u}^{2}\right)\left(m_{c}^{2}-m_{u}^{2}\right)\left(m_{b}^{2}-m_{s}^{2}\right)\left(m_{b}^{2}-m_{d}^{2}\right)\left(m_{s}^{2}-m_{d}^{2}\right),
$$

where $J \simeq 3 \times 10^{-5}$ is twice the area of the unitarity triangle. On the other hand the 4 generation counterpart is more complicated, but it can be simplified with the aid of phenomenological information and the assumption on the CKM matrix, $\left|V_{t d} V_{t^{\prime} d}^{*}\right|<<\left|V_{t i} V_{t^{\prime} i}^{*}\right|\left(i=s, b, b^{\prime}\right)$, as [4]

$$
\operatorname{Im} \operatorname{Tr}\left[M_{u} M_{u}^{\dagger}, M_{d} M_{d}^{\dagger}\right]^{3} \simeq-6 A_{s b}^{t t^{\prime}}\left(m_{t^{\prime}}^{2}-m_{t}^{2}\right) m_{t^{\prime}}^{2} m_{t}^{2} m_{b^{\prime}}^{4} m_{b}^{2}
$$


where $A_{s b}^{t t^{\prime}}=\operatorname{Im}\left(V_{t s}^{*} V_{t^{\prime} b}^{*} V_{t b} V_{t^{\prime} s}\right)$. One can observe that the mass factors lead to the huge enhancement of $\Delta(\omega)$, compared to the 3 generation case. This is in line with the intuitive argument in 11 .

As discussed by HS, the perturbative expansion in the quark masses breaks down for heavy quarks, and the above result could be reduced due to a large $m_{b^{\prime}}$ if the perturbation is not used. Thus, the above result might overestimate the asymmetry from the d-type quarks. The u-type quark contribution, at leading order, is the same as Eq. (2) except the difference of the overall sign, hence, leading to a complete cancellation at this level. The above-mentioned remark is, however, also applied for the u-type quark contribution, and a possible amount of reduction would be even larger as it contains the contributions from the two heavy quarks, $t$ and $t^{\prime}$. Then, we simply neglect the u-type quark contribution in $\Delta(\omega)^{1}$.

Eventually, the baryon asymmetry generated during the EWPhT is

$$
\frac{n_{B}}{s} \sim 0.9 \times 10^{-10}\left(\frac{A_{s b}^{t \prime^{\prime}}}{4 \times 10^{-4}}\right)\left(\frac{m_{t^{\prime}}}{550 \mathrm{GeV}}\right)^{4}\left(\frac{m_{b^{\prime}}}{550 \mathrm{GeV}}\right)^{4},
$$

where $T=100 \mathrm{GeV}$ and $M_{W}(T)=50 \mathrm{GeV}$ are adopted, and $A_{s b}^{t t^{\prime}} \sim 10^{-4}$ is still compatible with flavor data [5]. Then, the generated baryon asymmetry seems to be in the right ballpark. Although this result should be semiquantitative at most, our finding would deserve further investigation. Note that $A_{s b}^{t t^{\prime}}$, related with $b \rightarrow s$ transition, could be measured at the LHC [6].

At this conference, CMS announced they excluded the SM4 Higgs for $120 \mathrm{GeV}<m_{h}<600$ $\mathrm{GeV}$ (95\% C.L.) [7]. CMS also put the mass bound $m_{t^{\prime}}>450 \mathrm{GeV}$ (95\% C.L) [7], in front of the unitarity bound. These suggest an interesting scenario where the electroweak symmetry breaking is triggered by pair condensations of $4 \mathrm{G}$ quarks, arising from the large Yukawa couplings and/or a new strong interaction. In such a case, the issue of the EWPhT becomes an open problem, and a rich spectrum of composite scalars may realize a strongly first-order EWPhT [8].

\section{References}

[1] W. -S. Hou, Chin. J. Phys. 47, 134 (2009).

[2] G. R. Farrar and M. E. Shaposhnikov, Phys. Rev. Lett. 70, 2833 (1993) [Erratum-ibid. 71, 210 (1993)].

[3] M. B. Gavela, P. Hernandez, J. Orloff and O. Pene, Mod. Phys. Lett. A 9, 795 (1994); P. Huet, E. Sather, Phys. Rev. D51, 379-394 (1995).

[4] W. S. Hou, Y. Y. Mao and C. H. Shen, Phys. Rev. D 82, 036005 (2010).

[5] W. -S. Hou, C. -Y. Ma, Phys. Rev. D82, 036002 (2010).

[6] W. -S. Hou, M. Kohda, F. Xu, [arXiv:1107.2343 [hep-ph]].

[7] Plenary talk by G. Tonelli at this conference.

[8] Y. Kikukawa, M. Kohda, J. Yasuda, Prog. Theor. Phys. 122, 401-426 (2009).

\footnotetext{
${ }^{1}$ There is another type of major contribution to $\Delta(\omega)$, called $\Delta_{7}$ in HS, which arises when the finite quark masses in the broken phase are taken into account in the self-energy. In the 3 generation case $\Delta_{7}$ gives larger contribution to the BAU than Eq. 22, while we confirmed that in the 4 generation case the contribution from Eq. 2) dominates.
} 